\title{
Weyl law for contractive maps
}

\author{
María E. Spina, Alejandro M. F. Rivas, and Gabriel G. Carlo \\ Departamento de Física, CNEA, Libertador 8250, (C1429BNP) Buenos Aires, Argentina
}

(Dated: March 15, 2013)

\begin{abstract}
We find the Weyl law followed by the eigenvalues of contractive maps. An important property is that it is mainly insensitive to the dimension of the corresponding invariant classical set, the strange attractor. The usual explanation for the fractal Weyl law emergence in scattering systems (i.e., having a projective opening) is based on classical phase space distributions evolved up to the quantum to classical correspondence (Ehrenfest) time. In the contractive case this reasoning fails to describe it. Instead, we conjecture that the support for this behavior is essentially given by the strong non-orthogonality of the eigenvectors of the contractive superoperator.
\end{abstract}

PACS numbers: 05.45.Mt, 03.65.Sq, 05.45.Df

\section{INTRODUCTION}

The study of open quantum systems has recently become a very active field [1]. The reasons are many, including the development of quantum information and computation 2, 3], quantum optics and scattering systems [4, 5]. Particularly in this latter case the fractal Weyl law has been proposed. This law predicts the way in which the long-lived resonances of these systems grow as a function of $\hbar$. The fundamental ingredient is the classical invariant set, which in this kind of systems is the repeller, i.e., the set of trajectories non-escaping in the past and in the future. In fact, this law says that the number of long-lived quasibound states is proportional to $\hbar^{-\left(1+d_{H}\right)}$, where $d_{H}$ is the partial Hausdorff dimension of the repeller [ [6].

There is a vast literature that has contributed to gain confidence on this conjecture by means of numerical tests conducted on many systems [7]. However, open quantum maps have been the main tool in these studies, as they offer great simplicity in the calculations without losing much generality [8 10]. For them, the fractal Weyl law predicts that the resonances grow as $\hbar^{-d}$, where $d$ is the partial fractal dimension of the repeller. But if the way to open the system is nonprojective the available literature is very scarce. Recently [11] this situation has been analyzed for dissipative quantum operations that can be thought as a phase space contraction leading to dissipative dynamics [12]. In that work a dissipative baker map has been studied, where all classical initial conditions asymptotically fall on a strange attractor. The quantum counterpart has been implemented by means of a noise superoperator written in terms of Kraus operators [13]. The number of long-lived resonances has been found to behave in a rather different way compared to the usual prediction of the fractal Weyl law. In fact, this number grows as a power law in $\hbar$, but the exponent is mainly insensitive to the dimension of the fractal invariant set.

In this work we analyze this behavior in depth. We find the Weyl law for the spectra of contractive noise. In order to explain its emergence and discrepancies with the usual fractal Weyl law, we first follow the same steps than in the case of scattering systems (i.e., having a projec- tive opening). This is done in terms of an initial classical distribution (that in this case shrinks following the associated dissipation) evolved up to the quantum to classical correspondence time $T_{\mathrm{Ehr}}$, the Ehrenfest time. We propose a theoretical expression for this time based on dynamical considerations and confirm its validity by means of the exploration of the classical phase space distributions and the eigenvectors of the contractive superoperator. However, this reasoning does not lead to a satisfactory explanation. We conjecture that the strong nonorthogonality of the right eigenvectors is the main reason behind this behavior.

This paper is organized as follows: in Section II we briefly describe the dissipative model that we have used and give the expression of the Weyl law for the contractive baker map. In Section [II] the numerical results are analyzed and we explore possible explanations for the emergence of the here obtained Weyl law supported by studies of the phase space distributions and the properties of eigenvectors. Finally, we give our conclusions in Section IV

\section{THE WEYL LAW FOR CONTRACTIVE MAPS}

As in our previous work [11] we have investigated the spectral behavior of the dissipative baker map, which is defined on the 2 -torus $\mathcal{T}^{2}=[0,1) \times[0,1)$ by

$$
\mathcal{B}(q, p)= \begin{cases}(2 q, \epsilon p / 2) & \text { if } 0 \leq q<1 / 2 \\ (2 q-1,(\epsilon p+1) / 2) & \text { if } 1 / 2 \leq q<1\end{cases}
$$

Besides contracting the torus in the $p$ direction by a $\epsilon$ factor, this map stretches the unit square by a factor of two in the $q$ direction, squeezes it by the same factor in the $p$ direction, and then stacks the right half onto the left one. As a result a strange attractor sets in after a few time steps regardless of the nature of the initial condition.

The first step to quantize it is to impose on any state $|\psi\rangle$ periodic boundary conditions on the torus, for both the position and momentum representations. Then, 
we take $\langle q+1 \mid \psi\rangle=e^{i 2 \pi \chi_{q}}\langle q \mid \psi\rangle$, and $\langle p+1 \mid \psi\rangle=$ $e^{i 2 \pi \chi_{p}}\langle p \mid \psi\rangle$, with $\chi_{q}, \chi_{p} \in[0,1)$. There is a finite dimension $N=(2 \pi \hbar)^{-1}$ for the corresponding Hilbert space and a discrete set of position and momentum eigenstates, which is given by $\left|q_{j}\right\rangle=\left|\left(j+\chi_{q}\right) / N\right\rangle(j=0,1, \ldots N-1)$, and $\left|p_{k}\right\rangle=\left|\left(k+\chi_{p}\right) / N\right\rangle(k=0,1, \ldots N-1)$, whose eigenvalues are $q_{j}, p_{k}$. A discrete Fourier transform, i.e.

$$
\left(G_{N}\right)_{k j} \equiv\left\langle p_{k} \mid q_{j}\right\rangle=\frac{1}{\sqrt{N}} \exp \left(\frac{-i 2 \pi}{N}\left(j+\chi_{q}\right)\left(k+\chi_{p}\right)\right)
$$

relates these sets. We take anti-symmetric boundary conditions, this meaning $\chi_{q}=\chi_{p}=1 / 2$. For an even $N$ dimensional Hilbert space, the quantum baker map is defined in the momentum representation as [14, 15]

$$
B_{N}=\left(\begin{array}{cc}
G_{N / 2} & 0 \\
0 & G_{N / 2}
\end{array}\right) G_{N}^{-1}
$$

with $B_{N}$ a unitary matrix (closed quantum baker map).

We introduce dissipation by means of a non-unital quantum operation [12] implemented by an $N^{2} \times N^{2}$ Kraus superoperator of the form:

$$
M=\sum_{\mu=0}^{N-1} A^{\mu} \otimes A^{\mu \dagger} .
$$

Quantum operations act on the density matrix, $\otimes$ denotes the place where this later must be inserted in order to implement the corresponding quantum operation. Here

$$
A^{\mu}=\sum_{i=\mu}^{N-1} \sqrt{\left(\begin{array}{c}
i \\
i-\mu
\end{array}\right) \epsilon^{i-\mu}(1-\epsilon)^{\mu}}\left|p_{i-\mu}\right\rangle\left\langle p_{i}\right|
$$

are operators that induce transitions towards the momentum state $\left|p_{i=0}\right\rangle$. The coupling constant $\epsilon$ has the same value as the dissipation parameter of the corresponding classical map. $M$ is a trace preserving $\left(\sum_{\mu} A_{\mu}^{\dagger} A_{\mu}=1\right)$ and non-unital $\left(\sum_{\mu} A_{\mu} A_{\mu}^{\dagger} \neq 1\right)$ superoperator, which describes a process contracting phase space volume. The complete quantum dissipative dynamics is obtained by composing $M$ with the unitary map (2),

$$
\$=\left(B_{N} \otimes B_{N}^{\dagger}\right) \circ M .
$$

In this work we have computed the eigenvalue spectrum of superoperoperator (5) for different values of the contraction parameter $(\epsilon=0.8,0.7,0.6,0.4)$ and of the dimension $(90 \leq N \leq 180)$. For each case we have counted the number of complex eigenvalues $\lambda$ (with $\left.|\lambda|=\exp \left(\frac{-\gamma}{2}\right)\right)$ with a decay rate $\gamma$ smaller than a given value $\gamma_{\text {cut }}$. The data are collected in Fig. 1 which displays the fraction of resonances $f_{\text {long-lived }}$ as a function of $\epsilon, N$ and the cut-off value $\gamma_{\text {cut }}$ (in a wide range $\left.2 \leq \gamma_{\text {cut }} \leq 14\right)$.

By fitting these numerical results, we obtain a remarkably compact and simple expression:

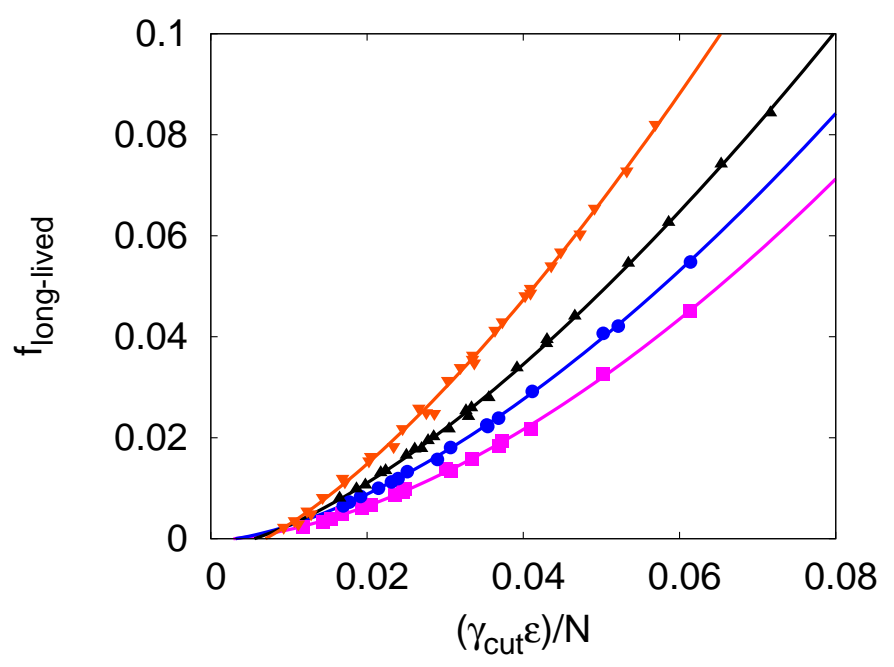

FIG. 1: (Color online) Weyl law for contractive noise:

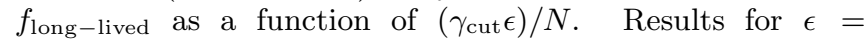
$0.8,0.7,0.6$ and 0.4 are represented by means of up triangles (in red), down triangles (in black), dots (in blue) and squares (in magenta), respectively.

$$
f_{\text {long-lived }}\left(\epsilon, \gamma_{\text {cut }}, N\right)=\frac{N_{\gamma<\gamma_{\text {cut }}}}{N^{2}}=C\left(\epsilon \gamma_{\text {cut }}\right)^{2 \nu}\left(N^{2}\right)^{-\nu}
$$

The values of $C$ and $\nu$, for four different values of $\epsilon$, are given in Table I. In the fourth column we display the semiclassical prediction $\nu_{\mathrm{sc}}$, which will be analyzed in Section III

TABLE I: Values of the fitted coefficients $C$ (column two) and $\nu$ (column three) for different values of $\epsilon$. The fourth column displays the semiclassical prediction $\nu_{\mathrm{sc}}$ described in Section III

\begin{tabular}{|l||l|l|l|}
\hline$\epsilon=0.8$ & $C=5.3$ & $\nu=0.72$ & $\nu_{\mathrm{sc}}=0.24$ \\
\hline$\epsilon=0.7$ & $C=4.4$ & $\nu=0.76$ & $\nu_{\mathrm{sc}}=0.34$ \\
\hline$\epsilon=0.6$ & $C=4.7$ & $\nu=0.79$ & $\nu_{\mathrm{sc}}=0.42$ \\
\hline$\epsilon=0.4$ & $C=5.3$ & $\nu=0.85$ & $\nu_{\mathrm{sc}}=0.57$ \\
\hline
\end{tabular}

These findings generalize the ones obtained in [11]. On the one hand they confirm the existence of a power law dependence of $\frac{N_{\gamma<\gamma_{\text {cut }}}}{N^{2}}$ on $N$ with an exponent which, in a meaningful range of validity, is fairly insensitive to the value of the dissipation parameter $\epsilon$. On the other hand, they hint (within a precision of 20\%) on a very simple dependence of the prefactor with both $\epsilon$ and the cut-off value $\gamma_{\text {cut }}$. We will leave the analysis of this prefactor, which is in general believed to be system-dependent, for future work [18] and concentrate in the following on the 
scaling of $\frac{N_{\gamma<\gamma_{\text {cut }}}}{N^{2}}$ with $N$. We will seek for an expression of $\nu$, in order to determine to which extent this exponent can be related to the underlying classical dynamics. For this we will follow an approach analogous to the one used in the formulation of the fractal Weyl law for chaotic maps with a projective opening [10] and discuss its limitations in the case of a contractive noise.

\section{CLASSICAL AND QUANTUM SUPPORT FOR THE EIGENVALUE STATISTICS}

A heuristic formulation of the fractal Weyl law for chaotic maps with a projective opening is based on the assumption that the number of long-lived resonances (associated with the classical repeller) scales as the volume of the evolved initial classical distribution up to the Ehrenfest time, that is, the volume of a finite (Ehrenfest)time repeller [10]. This volume can be calculated by a combination of two exponential laws that relate the probability to reside in the system (non escaping trajectories) and the quantum to classical correspondence.

In the case of a contractive noise the connection between the long-lived resonances and the structure of the classical invariant also exists. In particular we have verified in [11] that the Husimi representation of the projector corresponding to the eigenfunctions with slow escape rate concentrates on the phase space region corresponding to the classical strange attractor. It seems then natural to generalize the considerations usually applied to chaotic maps with a projective opening to the contractive case and investigate whether this scheme succeeds in accounting for the Weyl law of eq.(6). Our starting point will be the following relation [16]:

$$
f_{\text {long-lived }}\left(\epsilon, \gamma_{\text {cut }}, N\right) \sim A_{\text {clas }}^{2}
$$

between the fraction of long-lived resonances and the volume of the attractor $A_{\text {clas }}$ which shrinks exponentially until the Ehrenfest time according to:

$$
A_{\text {clas }}=\exp -\left(\gamma_{\mathrm{cl}} T_{\mathrm{Ehr}}\right)
$$

Notice in eq. (17) the square (instead of linear) dependence on $A_{\text {clas }}$, which is due to the use of the superoperoperator formalism to model the contractive noise. The classical decay rate $\gamma_{\mathrm{cl}}$ and the correspondence (Ehrenfest) time $T_{\mathrm{Ehr}}$ are then the two main ingredients of this approach that should be evaluated.

The classical decay rate can be easily calculated by following the time evolution of a uniform distribution in phase space under the action of dissipation. It is straightforward to see that after t time steps the original distribution will occupy $2^{t}$ fringes in the $q$ direction, each fringe having a width $\left(\frac{\epsilon}{2}\right)^{t}$. Hence the total phase space area occupied by the distribution as a function of time is $A_{\text {clas }} \equiv e^{-t \gamma_{\mathrm{cl}}}=\epsilon^{t}$, and then the classical decay rate is given by $\gamma_{\mathrm{cl}}=-\ln \epsilon$.
Determination of the Ehrenfest time is a more subtle issue. Understood as the time at which the quantum and the classical descriptions differ, we can start our reasoning following the lines of what is done in the case of area preserving maps. In fact, there are two different ways to conceive this correspondence time. The first one is the time $T_{\text {Ehr1 }}$ at which a given initial semiclassical distribution (a coherent state of width $\sqrt{\hbar}$, for instance) spreads up to the border of the system along the unstable direction (manifold). This time is related to the expansive Lyapunov exponent $\lambda_{1}$, such that $T_{\mathrm{Ehr} 1} \propto \frac{\ln N}{\lambda_{1}}$. On the other hand, the time $T_{\mathrm{Ehr} 2}$ is that corresponding to the initial distribution shrinking along the stable direction to a size of the order of the Planck cell $(1 / N)$. This time is related to the contractive Lyapunov exponent $\lambda_{2}$, such that $T_{\mathrm{Ehr} 2} \propto \frac{\ln N}{\left|\lambda_{2}\right|}$. Of course, in the case of an area preserving map $\lambda_{1}+\lambda_{2}=0$ and both times coincide. However, under a contractive noise, our dissipative map gives $\lambda_{1}=\ln 2$ while $\lambda_{2}=-\ln \frac{2}{\epsilon}$. Hence, we propose the shortest $T_{\mathrm{Ehr} 2} \propto \ln N / \ln \frac{2}{\epsilon}$ as the global quantum to classical correspondence time for this map.

In order to verify this assumption, we have numerically estimated the correspondence time. This can be accomplished quite easily by evaluating the overlap $O_{\mathrm{cl}-\mathrm{q}}$ between the finite time classical attractor and the Husimi distribution of a uniform initial state evolved up to the same time. If we exploit the fact that for the baker map the interesting features of the distribution (namely its fractality) are only in the $p$ coordinate we can notably simplify this task. In fact, we just calculate the norm of the evolved wavefunction, restricted to the region occupied by the classical distribution at any given time. As a result we have obtained Fig. 2 where these overlaps are shown as a function of the map iterations. We have found that, besides small fluctuations and the lack of precision inherent to the discrete time steps of the map, the results confirm our theoretical prediction (see vertical lines as a guide).

Inserting the expressions of $\gamma_{\mathrm{cl}}$ and $T_{\mathrm{Ehr} 2}$ in eq. (8) gives $A_{\text {clas }}=N^{-\nu_{\mathrm{sc}}}$, where $\nu_{\mathrm{sc}}=2-d$, and $d=$ $1+\ln (2) /(\ln (2)-\ln (\epsilon))$ is the fractal dimension of the classical attractor. The values of the semiclassical $\nu_{\mathrm{sc}}$ are listed in the fourth column of Table [ showing a dramatic discrepancy with the values obtained by fitting our numerical results with eq. (6). Besides an overall factor of $\sim 2$ between $\nu$ and $\nu_{\mathrm{sc}}$, the semiclassical exponent shows a dependence on $\epsilon$ (via the fractal dimension of the attractor) which is absent in the fitted $\nu$ which are practically constant. Then, it becomes clear that the way of reasoning that has provided with a reasonable explanation for the emergence of the usual fractal Weyl law for systems subjected to projective noise can no longer be applied to contractive dynamics. We are now faced with the question of where this discrepancy comes from.

At the basis of eq.(17) is the assumption that the number of long-lived quantum states can be approximated by the number of Planck cells which fit into the phase space volume of the classical invariant set. This, in turn, 

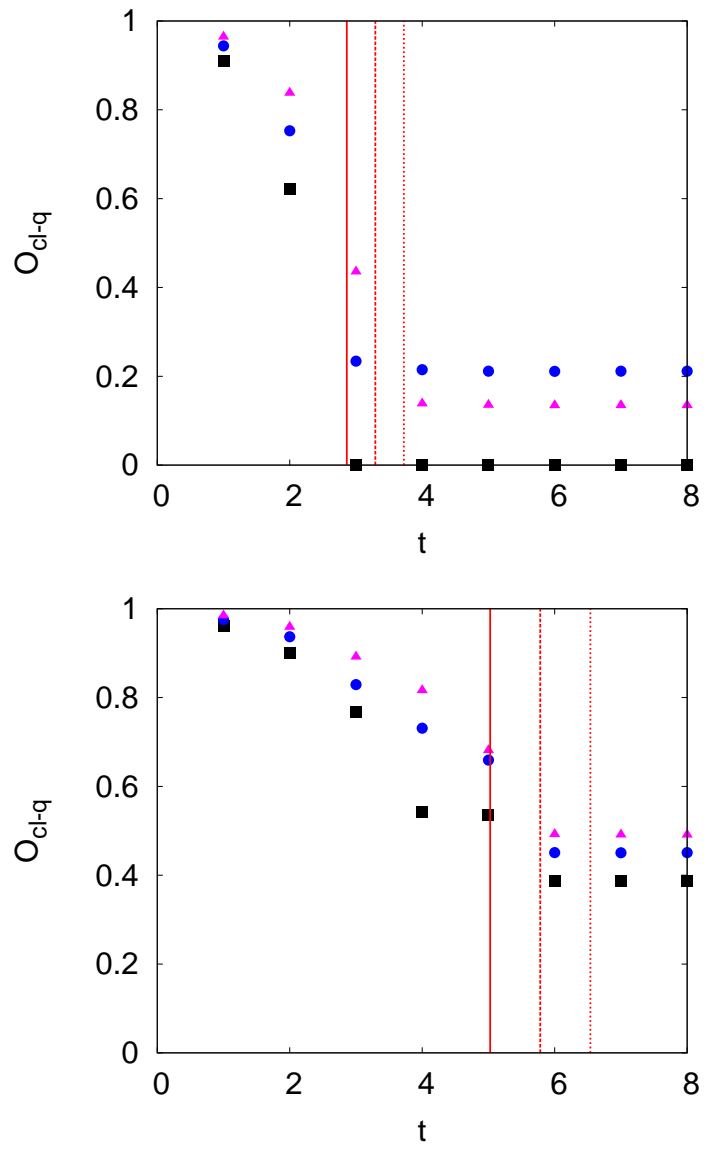

FIG. 2: (color online) Overlap between the phase space region occupied by the quantum and classical attractors as a function of time $t$ (map iterations). Upper panel corresponds to $\epsilon=$ 0.4 , lower panel to $\epsilon=0.8$. Results for $N=100,200$, and 400 are represented with squares (in black), dots (in blue) and up triangles (in magenta), respectively. Vertical lines show the corresponding theoretical values of $T_{\mathrm{Ehr} 2}$ for $N=100,200$, and 400. (full, dashed and dotted lines, respectively).

supposes that to a good approximation the eigenfunctions supported by this set are non-overlapping. Even though we cannot strictly speak of orthogonality, since the operators describing open systems are not normal, we know that in the case of projective openings the long-lived eigenfunctions are quasi-orthogonal (while the short-lived ones present a high degree of degeneracy). This explains the success of the fractal Weyl law in the projective case. In the case of contractive dynamics we will investigate this point by defining the overlap matrix $P_{i j}=\operatorname{Tr}\left(R_{i}^{\dagger} R_{j}\right)$, where $R_{i}$ are the right eigenstates corresponding to the superoperator $\$$ of Eq. (5) (this is not to be confused with the biorthogonality of the right and left eigenfunctions of a superoperator, which states that $\left.\operatorname{Tr}\left(L_{i}^{\dagger} R_{j}\right)=\delta_{i, j}\right)$. The overlap matrix elements corresponding to the contractive map with $N=180$ and $\epsilon=0.4,0.6,0.8$ for the 200 longest-lived eigenstates are displayed in panels (a), (b), and (c) of Fig. 3, respectively. A grayscale is used to represent them, going from white corresponding to value 0 to black corresponding to the maximum values. We observe that the off-diagonal elements are clearly non negligible. Moreover, their value grows with the contractive power of the corresponding map (as $\epsilon$ decreases). For comparison we show in panel (d) the overlap matrix for a projective case, obtained by opening the baker map along two symmetric bands in the q-direction, of width $\delta p=0.1$ and centered at $p=0$ and $p=N-1$. In this case, as expected, the matrix is almost diagonal.

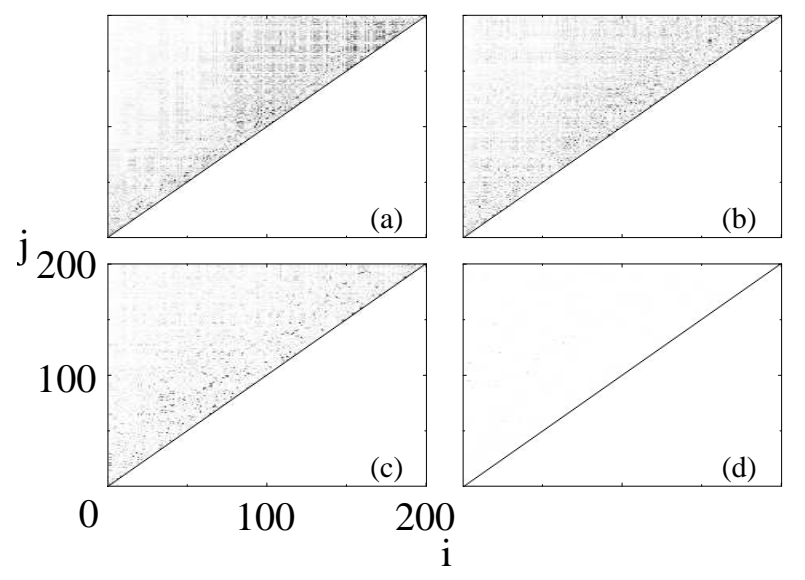

FIG. 3: Overlap matrices $P_{i j}$ of the first 200 right eigenstates with $N=180$ (ordered by decreasing modulus of the eigenvalues) corresponding to the contractive map for $\epsilon=0.4,0.6$, and 0.8, (panels (a), (b), and (c), respectively). For comparison we show the same overlap matrix but for a projective opening that amounts to 0.2 of the phase space. Only the upper half of the matrices is shown.

The different degree of non-orthogonality of the longlived resonances in both models is also reflected in the phase space distribution of these states. In panel a) and c) of Fig. 4 we show the sum up to $\gamma_{\text {cut }}$ of the Husimi representation of the longest-lived right eigenstates:

$$
\sum_{\gamma=0}^{\gamma_{\text {cut }}} \frac{\left\langle z\left|R_{\gamma}\right| z\right\rangle\left\langle z\left|R_{\gamma}^{\dagger}\right| z\right\rangle}{\left\langle R_{\gamma} \mid R_{\gamma}^{\dagger}\right\rangle},
$$

with $\left\langle z\left|R_{\gamma}\right| z\right\rangle=\operatorname{Tr}\left(R_{\gamma}^{\dagger},|z\rangle\langle z|\right)$ where $|z\rangle$ are coherent states centered at $z=(q, p)$. Panels b) and d) display the analogous sum (9) corresponding to the Husimi representation but of the Schur eigenvectors, which constitute the orthogonal basis associated with the eigenvalues $\lambda$ with $|\lambda| \geq \exp \left(\frac{-\gamma_{\text {cut }}}{2}\right)$.

In the case of the contractive map (upper line) we observe that the area of phase space occupied by the sum of the Husimi distributions is smaller than the area corresponding to the subspace spanned by the Schur decomposition. This is a clear sign of the non-orthogonality of the eigenstates for this kind of superoperators [17]. 
On the contrary, the lower panels (c) and (d) show that for the case of a projective opening both distributions look much the same, indicating that the assumption of quasi-orthogonality for the long-lived eigenfunctions is justified.

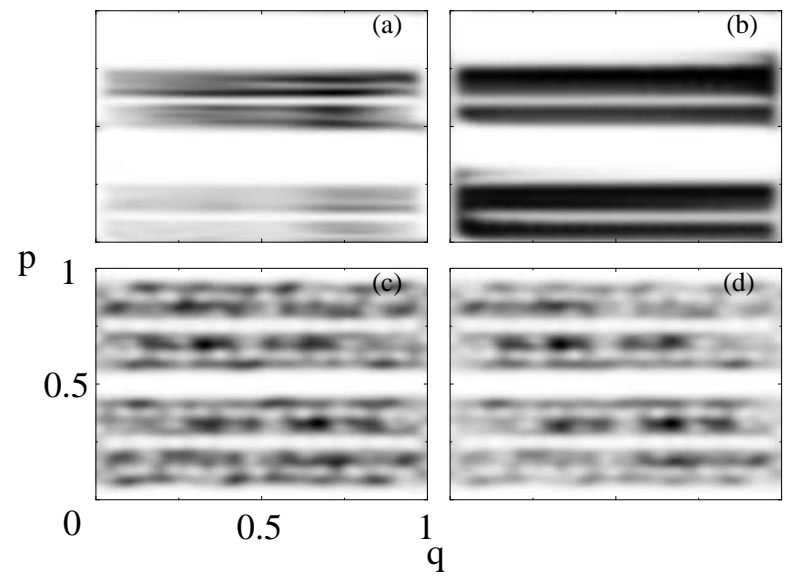

FIG. 4: In panel (a) we show the sum (9) of the Husimi representation corresponding to the first 600 right-right eigenvectors for the contractive map at $\epsilon=0.6$ for $N=180$. Panel (b) displays the analogous sum corresponding to the Husimi representation of the Schur eigenvectors. For comparison, in the lower panels (c) and (d) we show the same distributions than in panels (a) and (b) respectively but for the projective opening case of Fig. 3 (d).

\section{CONCLUSIONS}

We have found an expression of the Weyl law for the spectra of the contractive baker map. An analogous sim- ple dependence on $\left(\gamma_{\text {cut }} \epsilon\right) / N$ has also been obtained for a dissipative kicked top map on the sphere. We were not able to explain the emergence of this law by means of the usual line of reasoning applied to the projective case. Very simply put, the idea is counting resonances. This has been traditionally accomplished by partitioning the phase space volume occupied by a finite time classical invariant set (the repeller). In fact, implies a pseudo orthogonality of the long-lived eigenstates. We could verify that this is indeed the case for the projectively opened baker map, a system that has been paradigmatically used in the fractal Weyl law literature. But when it comes to the dissipative baker map used in this work, we have clearly identified a high degree of non-orthogonality. This is the main reason behind the failure of the usual reasoning for explaining the emergence of the Weyl law.

As a result, we think that a new method for counting the long-lived resonance other than just partitioning the corresponding volume in phase space into Planck cells, is the key to understand the statistical behavior of contractive maps. In the future, we hope to find a theoretical explanation for it, including the one of the prefactor and the dependence on $\epsilon$ and $\gamma_{\text {cut }}$ [18].

\section{Acknowledgments}

Support from CONICET is gratefully acknowledged.
[1] U. Weiss, Quantum Dissipative Systems (World Scientific, Singapore, 2008).

[2] M.A. Nielsen and I.L. Chuang, Quantum Computation and Quantum Information, Cambridge University Press (2000).

[3] J. Preskill, Lecture Notes for Physics 229: Quantum Information and Computation, http://www . theory. caltech.edu/people/preskill/ph229/.

[4] S. Oberholzer, E.V. Sukhorukov and C. Schönenberger, Nature 415, 765 (2002); W. Lu, Z. Ji, L. Pfeiffer, K.W. West and A.J. Rimberg, Nature 423, 422 (2003); J. Bylander, T. Duty and P. Delsing, Nature 434, 361 (2005).

[5] C. Gmachl, F. Capasso, E.E. Narimanov, J.U. Nöckel, A. D. Stone, J. Faist, D.L. Sivco and A.Y. Cho, Science 231, 486 (1998); S.-Y. Lee, S. Rim, J.-W. Ryu, T.-Y. Kwon, M. Choi and C.-M. Kim, Phys. Rev. Lett. 93, 164102 (2004); T. Tanaka, M. Hentschel, T. Fukushima and T. Harayama, Phys. Rev. Lett. 98, 033902 (2007).

[6] W.T. Lu, S. Sridhar and M. Zworski, Phys. Rev. Lett.
91, 154101 (2003).

[7] J.A. Ramilowski, S.D. Prado, F. Borondo and D. Farrelly, Phys. Rev. E 80, 055201(R) (2009); A. Eberspacher, J. Main and G. Wunner, Phys. Rev. E 82, 046201 (2010).

[8] M. Novaes, arXiv:1211.724885v1 [quant-ph].

[9] S. Nonnenmacher, Nonlinearity 24, R123 (2011).

[10] S. Nonnenmacher and M. Rubin, Nonlinearity 20, 1387 (2007); D. L. Shepelyansky, Phys. Rev. E 77, 015202(R) (2008).

[11] G. G. Carlo, A. M. F. Rivas, and M. E. Spina, Phys. Rev. E 84, 066201 (2011).

[12] I.Garcia-Mata, M. Saraceno, M. E. Spina and G.Carlo, Phys. Rev. A 72, 062315 (2005).

[13] K. Kraus, States, Effects and Operations (SpringerVerlag, Berlin, 1983).

[14] M. Saraceno, Ann. Phys. 199, 37 (1990); M. Saraceno and R. O. Vallejos, Chaos 6, 193 (1996); A. Łoziński, P. Pakoński and K. Życzkowski, Phys. Rev. E 66, 065201(R) (2002). 
[15] M. Saraceno and A. Voros, Physica D 79, 206 (1994).

[16] A. Ishii, A. Akaishi, A. Shudo, and H. Schomerus Phys. Rev. E 85, 046203 (2012); H. Schomerus and J. Tworzydlo, Phys. Rev. Lett. 93, 154102 (2004);
[17] M.Kopp and H.Schomerus Phys. Rev. E 81, 026208 (2010).

[18] M.E. Spina, A.M.F. Rivas, and G.G. Carlo, in progress. 